\title{
A system for probing Casimir energy corrections to the condensation energy
}

\author{
Diego Pérez-Morelo (10) ${ }^{1,2,3}$, Alexander Stange (iD), Richard W. Lally ${ }^{4}$, Lawrence K. Barrett ${ }^{4}$, Matthias Imboden (1) \\ Abhishek Som ${ }^{6}$, David K. Campbell $\mathbb{B}^{1,4,6}$, Vladimir A. Aksyuk $\mathbb{B}^{2}$ and David J. Bishop ${ }^{1,4,6,7,8}$
}

\begin{abstract}
In this article, we present a nanoelectromechanical system (NEMS) designed to detect changes in the Casimir energy. The Casimir effect is a result of the appearance of quantum fluctuations in an electromagnetic vacuum. Previous experiments have used nano- or microscale parallel plate capacitors to detect the Casimir force by measuring the small attractive force these fluctuations exert between the two surfaces. In this new set of experiments, we aim to directly detect the shifts in the Casimir energy in a vacuum due to the presence of the metallic parallel plates, one of which is a superconductor. A change in the Casimir energy of this configuration is predicted to shift the superconducting transition temperature $\left(T_{c}\right)$ because of the interaction between it and the superconducting condensation energy. In our experiment, we take a superconducting film, carefully measure its transition temperature, bring a conducting plate close to the film, create a Casimir cavity, and then measure the transition temperature again. The expected shifts are smaller than the normal shifts one sees in cycling superconducting films to cryogenic temperatures, so using a NEMS resonator in situ is the only practical way to obtain accurate, reproducible data. Using a thin Pb film and opposing Au surface, we observe no shift in $T_{c}>12 \mu \mathrm{K}$ down to a minimum spacing of $\sim 70 \mathrm{~nm}$ at zero applied magnetic field.
\end{abstract}

\section{Introduction}

The Casimir force was first derived in 1948 by calculating the van der Waals force using retarded potentials ${ }^{1}$. This force is a purely quantum mechanical force that arises between two plates even when they are not electrically charged. Classically, there is no force on the plates. However, due to quantum fluctuations and the freezing out of the long-wavelength electromagnetic modes, there is a net pressure exerting an attractive force. Experimentally, the effect has been seen using a number of microscale systems and devices ${ }^{2-8}$. Reference ${ }^{4}$ discusses how the force varies with the metallic conductivity of the plates. References ${ }^{9,10}$ show how the effect can be used for

Correspondence: David J. Bishop (djb1@bu.edu)

'Department of ECE, Boston University, Boston, MA 02215, USA

${ }^{2}$ Physical Measurement Laboratory, National Institute of Standards and

Technology, Gaithersburg, MD 20899, USA

Full list of author information is available at the end of the article

These authors contributed equally: Diego Pérez-Morelo, Alexander Stange practical applications and refs. ${ }^{11,12}$ show how a repulsive force can be achieved. Additional work has also demonstrated the importance of nanopatterning ${ }^{13}$ and magnetic effects $^{14}$.

Given that the metallic conductivity changes the magnitude of the Casimir force, the question immediately comes to mind "what happens if the plates become superconducting?" The answer disappointingly is "not much." The Casimir effect averages the conductivity of the material over very large energy scales, while the superconducting gap is relevant only for the far infrared ${ }^{15}$. Therefore, while the effect of superconductivity is very large $(100 \%)$ on the DC conductivity, it is negligible and unmeasurable if averaged over the typical energy scales found in a Casimir cavity ${ }^{16}$. Therefore, one cannot see an effect on the measured Casimir force at the transition temperature $T_{\mathrm{c}}$.

However, as pointed out in work by Bimonte and coworkers $^{17}$, one might be able to see an effect of Casimir

\section{(c) The Author(s) 2020}

(c) (i) Open Access This article is licensed under a Creative Commons Attribution 4.0 International License, which permits use, sharing, adaptation, distribution and reproduction cc) in any medium or format, as long as you give appropriate credit to the original author(s) and the source, provide a link to the Creative Commons license, and indicate if changes were made. The images or other third party material in this article are included in the article's Creative Commons license, unless indicated otherwise in a credit line to the material. If material is not included in the article's Creative Commons license and your intended use is not permitted by statutory regulation or exceeds the permitted use, you will need to obtain permission directly from the copyright holder. To view a copy of this license, visit http://creativecommons.org/licenses/by/4.0/. 
energy on the superconductivity. In a type I superconductor, the critical parallel field $H_{\mathrm{c}||}(T)$ is given by the change in the free energy, $\Delta F$, which is the difference between the free energy in the superconducting and normal states:

$$
\left(H_{\mathrm{c} \|}(T)\right)^{2} \propto \Delta F(T)
$$

Reference ${ }^{17}$ suggests that this free energy change may also be related to the Casimir energy:

$$
\Delta F=E_{\text {cond }}(T)+\Delta E_{\text {Cas }}(T)
$$

where $E_{\text {cond }}(T)$ is due to the superconductivity and $\Delta E_{\text {Cas }}(T)$ is due to the Casimir energy. For small modulations in this Casimir term, the critical field is modulated by a factor proportional to the ratio $\Delta E_{\text {Cas }} /$ $E_{\text {cond. }}$ The calculations performed in ref. ${ }^{18}$ suggest that this fraction could be as large as $10 \%$ in certain thin-film materials with low condensation energies.

The theory suggests the following: first, placing a superconducting film in a Casimir cavity shifts $T_{\mathrm{c}}$. Second, measuring the effect is contingent upon keeping $E_{\text {cond }}$ constant. Therefore, any attempt to compare several different films, some inside cavities and some not, may suffer from uncertainty due to variations in the highly processdependent characteristics of superconducting thin films. Even on a single die, different regions of the deposited material may display slightly different superconducting transition temperatures due to thickness variations, local roughness, or temperature gradients during deposition. In this work, we present a technique in which a nanoelectromechanical system (NEMS) structure is used to move a plate relative to a single superconducting film in situ. The basic concept is shown in Fig. 1, in which we sit on the shoulder of the superconducting transition and actuate a nearby metallic plate, thus modulating the Casimir energy while monitoring the film resistance. Due to the sharp slope of the superconducting transition, a small change in $T_{\mathrm{c}}$ (due to a change in the Casimir energy) would manifest as a measurable change in the sample resistance. The present experimental approach can be extended to include the application of a magnetic field through the sample, allowing one to measure variations in the full $H_{\mathrm{c}}(T)$ curve for comparison with the existing theory, which currently does not provide quantitative predictions for $H=0^{17,18}$.

There are a number of experimental challenges posed by the concept shown in Fig. 1. According to calculations performed in refs. ${ }^{17,18}$, the spacing of the Casimir cavity should be in the range of a few nanometers to $100 \mathrm{~nm}$, and the film thickness should be on the order of $10 \mathrm{~nm}$. These characteristics place serious constraints on the choice of materials, many of which tend to ball up and form islanded microstructures when thin ${ }^{19}$. However, evaporating onto cryogenically cooled surfaces allows for the quenched condensation of the material, forming very smooth, amorphous films ${ }^{20,21}$. For this reason, an in situ deposition method is used in which the superconducting film is deposited at the chip-scale, below the superconducting transition temperature. This "fab-on-a-chip" methodology is explained further in the "Methods" section as well as in refs. ${ }^{21-25}$. It is with this quenchedcondensed thin film (which serves as one half of a tunable Casimir cavity) that we are able to probe changes in the Casimir energy.

\section{Results}

Experiments are performed by assembling a target chip and a source chip into a single package. Then, the system is cooled down to cryogenic temperatures, a superconducting

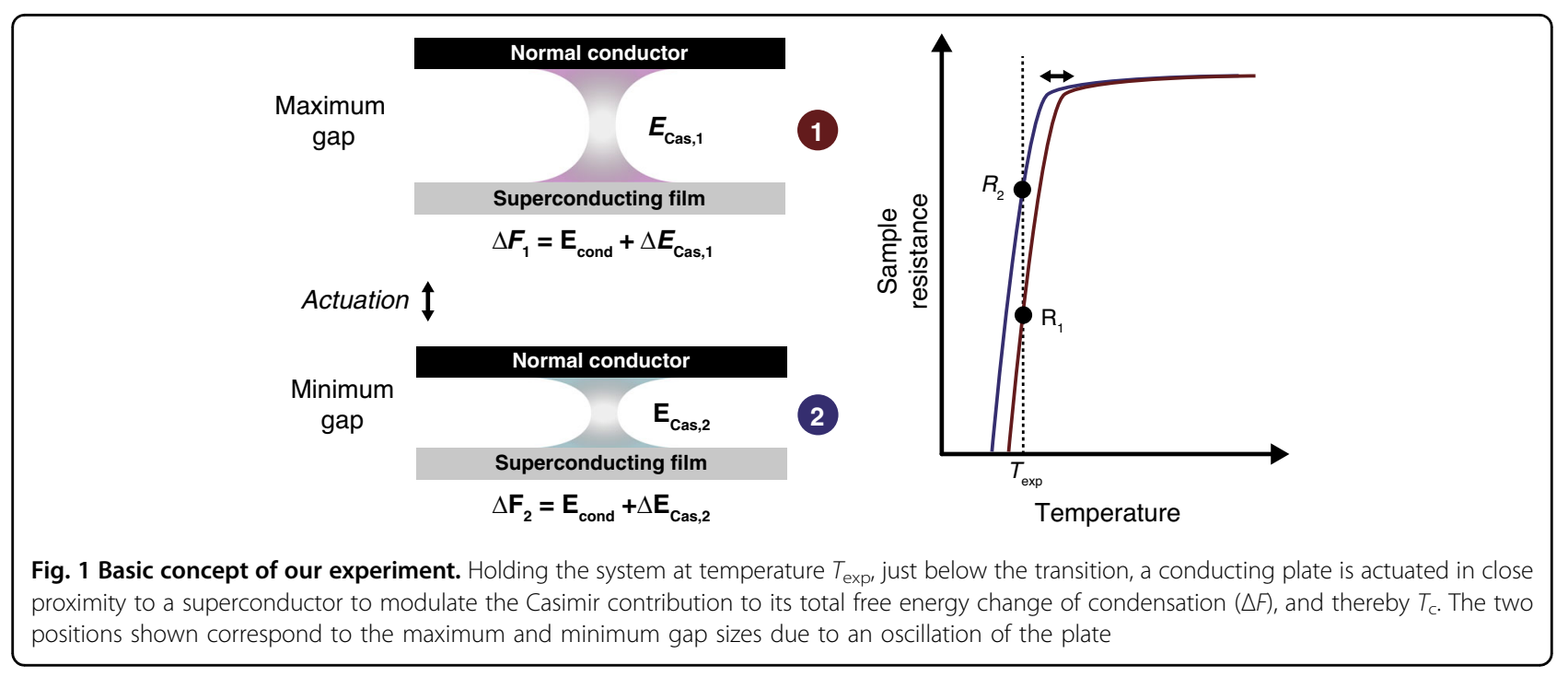


thin film is deposited onto the target die, and a Casimir cavity is formed. Next, the film is characterized, and the size of the Casimir cavity is dynamically tuned while monitoring the film resistance. The ex situ characterization of the film and cavity is also performed using scanning electron microscopy (SEM) and atomic force microscopy (AFM) after the experiment is complete. The following results are presented according to this sequence.

\section{Chip-scale evaporation and measurement setup}

The non-rigid Casimir cavity imposes serious nanofabrication challenges that must be overcome to successfully obtain a functional device. First, due to process incompatibilities, we need to be able to deposit the superconducting sample underneath the metallic plate after releasing the movable structures. Second, due to the oxidation of the thin film, evaporation and resistance measurements need to be performed without breaking vacuum conditions. Finally, low temperatures can be used to reduce the migration of the evaporated material along the substrate, enabling high-quality films.

Figure 2 shows a schematic of the three-die arrangement developed for this experiment, consisting of one NEMS target die (onto which the material will be evaporated) centered between two microsource dies. The flux of the material being evaporated diagonally from each microsource die will reach the target die and form a continuous, thin film underneath the top Au layer. This top Au layer on the target die serves as both a physical mask and a movable plate (to vary the Casimir gap size, which is nominally $g_{0}$ ). The bottom Au layer of the target die consists of two sets of electrical leads. The rectangular structures shown in Fig. 2 (target schematic) are the plate drive and sense electrodes for the movable gold plate, with a ground shield around them. The four leads heading off at $45^{\circ}(\mathrm{P} 1, \mathrm{P} 2, \mathrm{P} 3, \mathrm{P} 4)$ in the target die schematic are the four-point contacts to the superconducting film used to measure its resistance. The film is formed by two angular evaporations through the holes (red) that combine to form a continuous strip along the center. Another important feature of the target die is the presence of silicon oxide pillars that serve as physical stops for the movable Au plate, both protecting the sample from contact with the $\mathrm{Au}$ as well as providing information regarding the minimum cavity size achieved. The microsource dies, shown in purple, are separated by distance $d_{\text {sources }}$ and consist of an array of microscale heaters preloaded with a layer of superconducting material. The design and fabrication of the microsources and targets are explained further in the Supplementary Information.

\section{Quenched-condensed $\mathrm{Pb}$ thin film}

After the system is cooled to $\approx 3 \mathrm{~K}$, the microsources are slowly heated until the evaporation of $\mathrm{Pb}$ occurs. By applying short voltage pulses through microsources, very small amounts of material can be evaporated in a controlled manner. Because the target die is cooled to cryogenic temperatures, the $\mathrm{Pb}$ reaching the target forms a quenched-condensed film of just $20 \mathrm{~nm}$ to $30 \mathrm{~nm}$ (see ex situ AFM results). According to the mask pattern displayed in Fig. 2, the dual-angle deposited $\mathrm{Pb}$ film connecting four Au measurements leads to an " $\mathrm{H}$ " pattern, allowing for a four-point resistance measurement to be made.

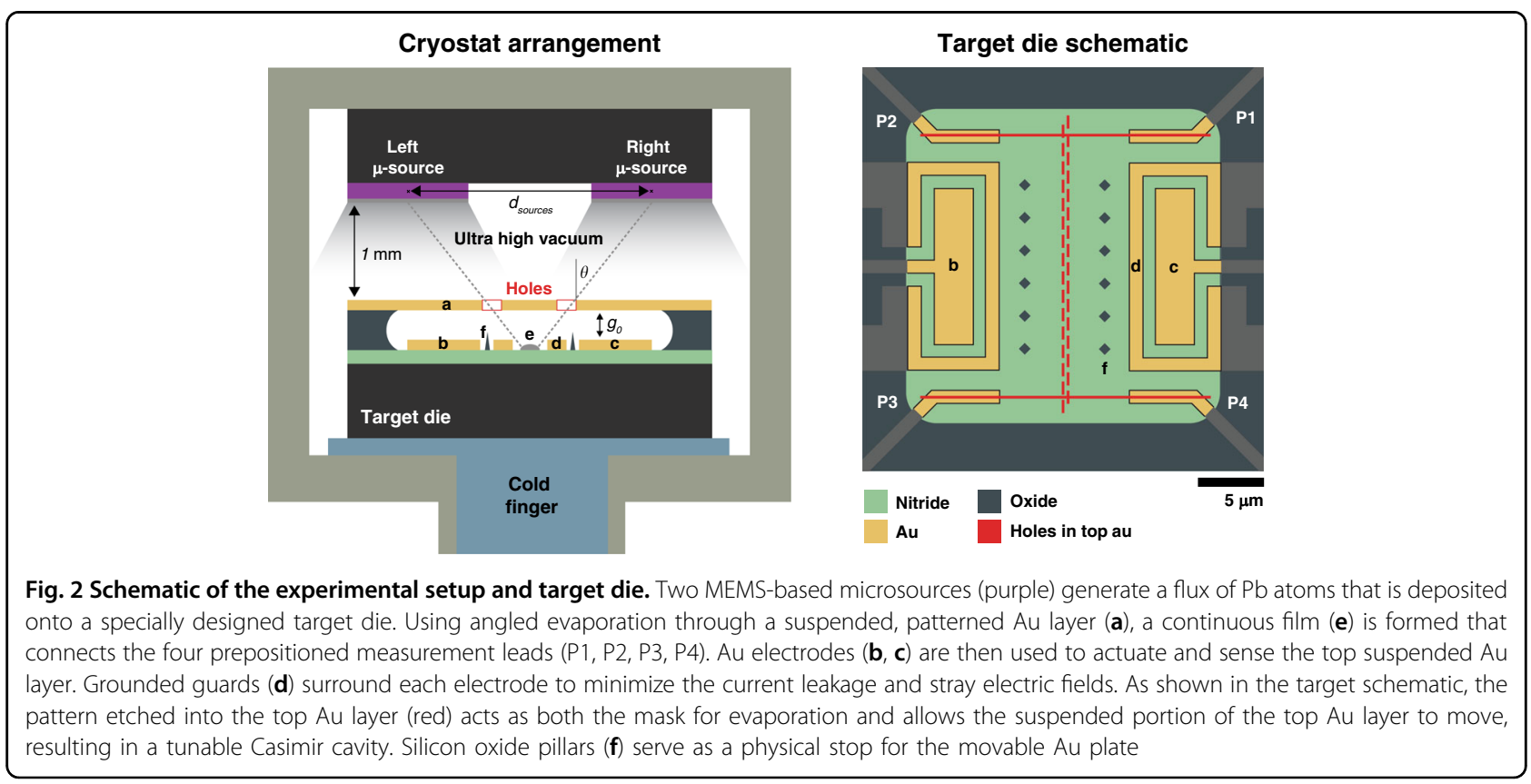




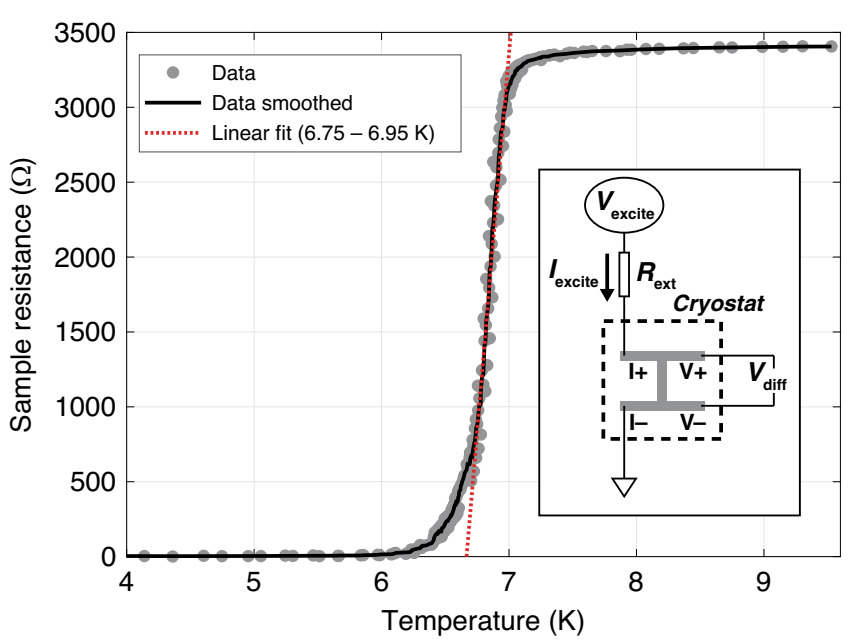

Fig. 3 Superconducting transition of the quenched-condensed Pb film. Data points (gray dots) are taken both sweeping up and down through the transition, and the black line is the smoothed average of these data points. The slope of the transition is calculated to be (11.4 \pm 0.4$)$ $\mathrm{k} \Omega / \mathrm{K}$. Inset: four-point resistance detection scheme. The current is applied through an external resistance, and the voltage drop across the Pb sample is measured

The measured resistance of the quenched-condensed $\mathrm{Pb}$ film as the temperature is swept from 4 to $9.5 \mathrm{~K}$ and back down is shown in Fig. 3. The temperature at which the film begins to condense into the superconducting phase is $\approx 7.05 \mathrm{~K}$, just under the bulk value of $7.193 \mathrm{~K}^{26}$, indicating a thicker film $\left(\sim 20 \mathrm{~nm}^{21}\right)$. The resistance of the film above $T_{\mathrm{c}}$ is $\approx 3.4 \mathrm{k} \Omega$, and the slope at the center of the transition is $(11.4 \pm 0.4) \mathrm{k} \Omega / \mathrm{K}$. The uncertainty on the slope is reported from the $95 \%$ confidence bounds of the linear fitting in the range of $6.75-6.95 \mathrm{~K}$.

\section{Cavity modulation and $T_{\mathrm{c}}$ shift measurement}

The experiment is carried out by setting the temperature of the cryostat to the steepest point of the transition $(T \approx 6.88 \mathrm{~K})$, where the measured resistance is most sensitive to changes in temperature. Then, the plate drive voltage is swept through its mechanical resonance while measuring the modulation of the resistance of the sample at the mechanical motion frequency. By operating the NEMS around resonance, we cannot only produce large changes in the gap size but also perform the measurement at a high frequency $(>1 \mathrm{MHz})$, which greatly reduces measurement noise. Plotted in Fig. 4 are three trials using this detection method, trial \#1, trial \#2, and trial \#3. The black data points show the frequency dependence of the amplitude and phase of the Au plate (left and right figures, respectively). A common Duffingnonlinear oscillator response is evident for amplitudes below the plate's mechanical contact with the oxide pillars. The blue data points show the change in resistance of the sample expressed in units of change in the transition temperature, using the slope calculated in Fig. 3.
The abscissa of the plots in Fig. 4 is the frequency of the signal being applied at the drive electrode. Because this is a purely AC signal, the electrostatic force applied to the plate and its resulting motion is actually at twice this frequency (see "Methods" section for details on this detection scheme). This $2 \times$ frequency difference between the electrical drive and the expected superconductor modulation signal largely eliminates any direct electrical crosstalk. For each trial, the frequency is swept upwards, and the plate amplitude/phase and sample resistance are all recorded simultaneously.

Additionally, the same three trials shown in Fig. 4 are plotted in Fig. 5 but are zoomed in to the region of interest (close to contact with the oxide pillars and just after the amplitude drop). In addition to plotting the data points, we include two sliding average curves to help visualize trends in the noisy data. These sliding averages take the mean of either one value or three values on either side of each data point (thin blue line and thick purple line, respectively).

\section{Ex situ characterization}

After running the experiment in the cryostat, the SEM and AFM measurements of the nanocavity (with the top $\mathrm{Au}$ surface removed) are performed. In Fig. 6a, the features of the bottom Au layer depicted in Fig. 2 are shown, consisting of two rectangular electrodes and four measurement leads. Additionally, the deposited $\mathrm{Pb}$ sample is visible, connecting all four leads and forming a continuous strip down the center. Figure $6 \mathrm{~b}$, c shows the AFM height measurements of the sample and the oxide stops. The sample height is measured to vary between 


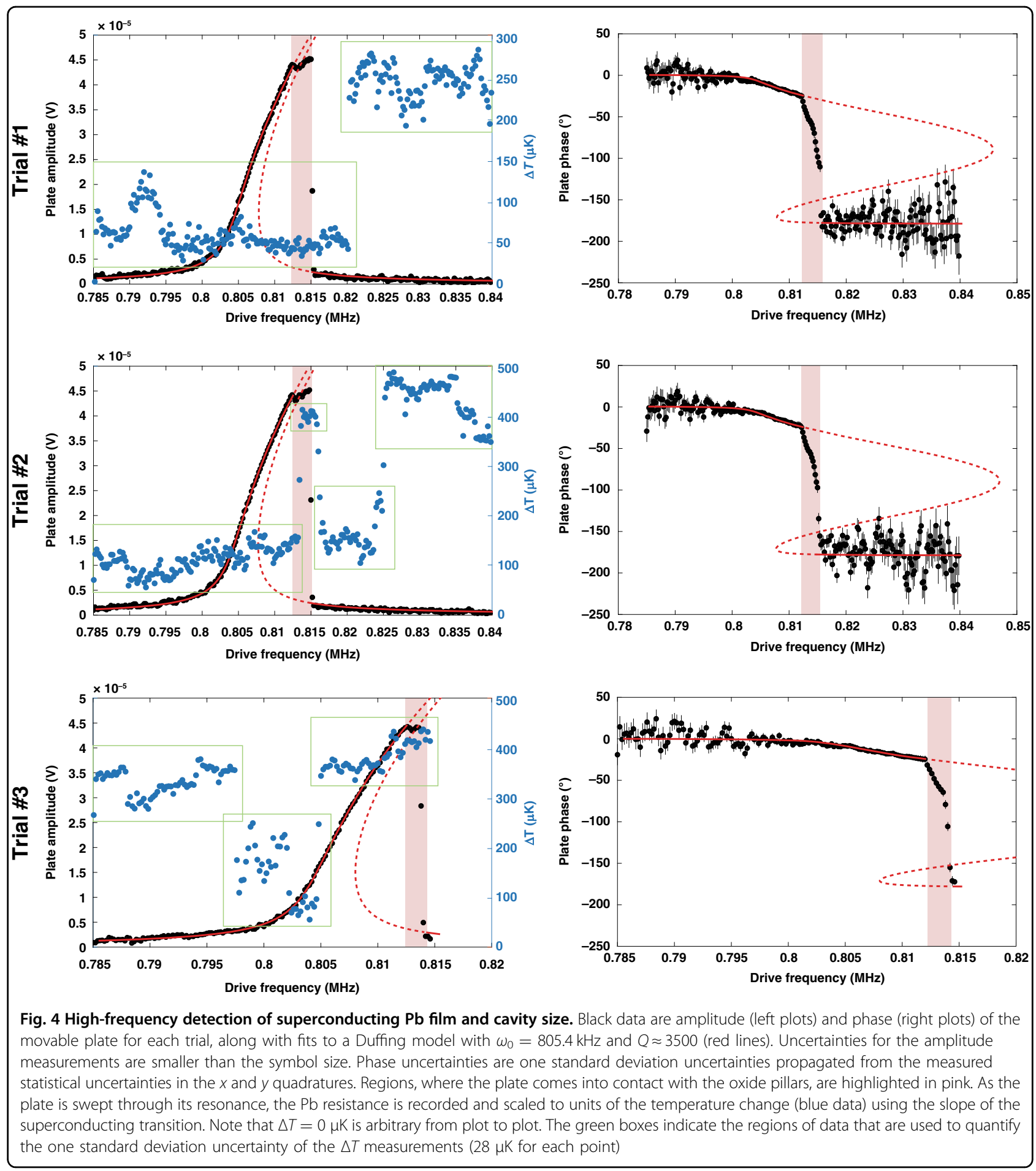

20 and $30 \mathrm{~nm}$. Using the SEM image, the length of the central portion of the sample is $(20.1 \pm 0.1) \mu \mathrm{m}$, and its width is $(403 \pm 57) \mathrm{nm}$. These values are obtained by averaging several measurements along the sample width and length, and the uncertainty is one standard deviation. The height of the oxide pillars is measured to be $160 \pm$ $1 \mathrm{~nm}$ from averaging the heights of the six measured pillars shown in Fig. 6b, and the uncertainty is one standard deviation.

Because the plate comes into contact with the oxide pillars, it is possible to estimate the vertical displacement of the center of the plate; however, to accomplish this, an assumption of the shape of the deformed Au must be made. The difficulty of this estimate is that the stress state 

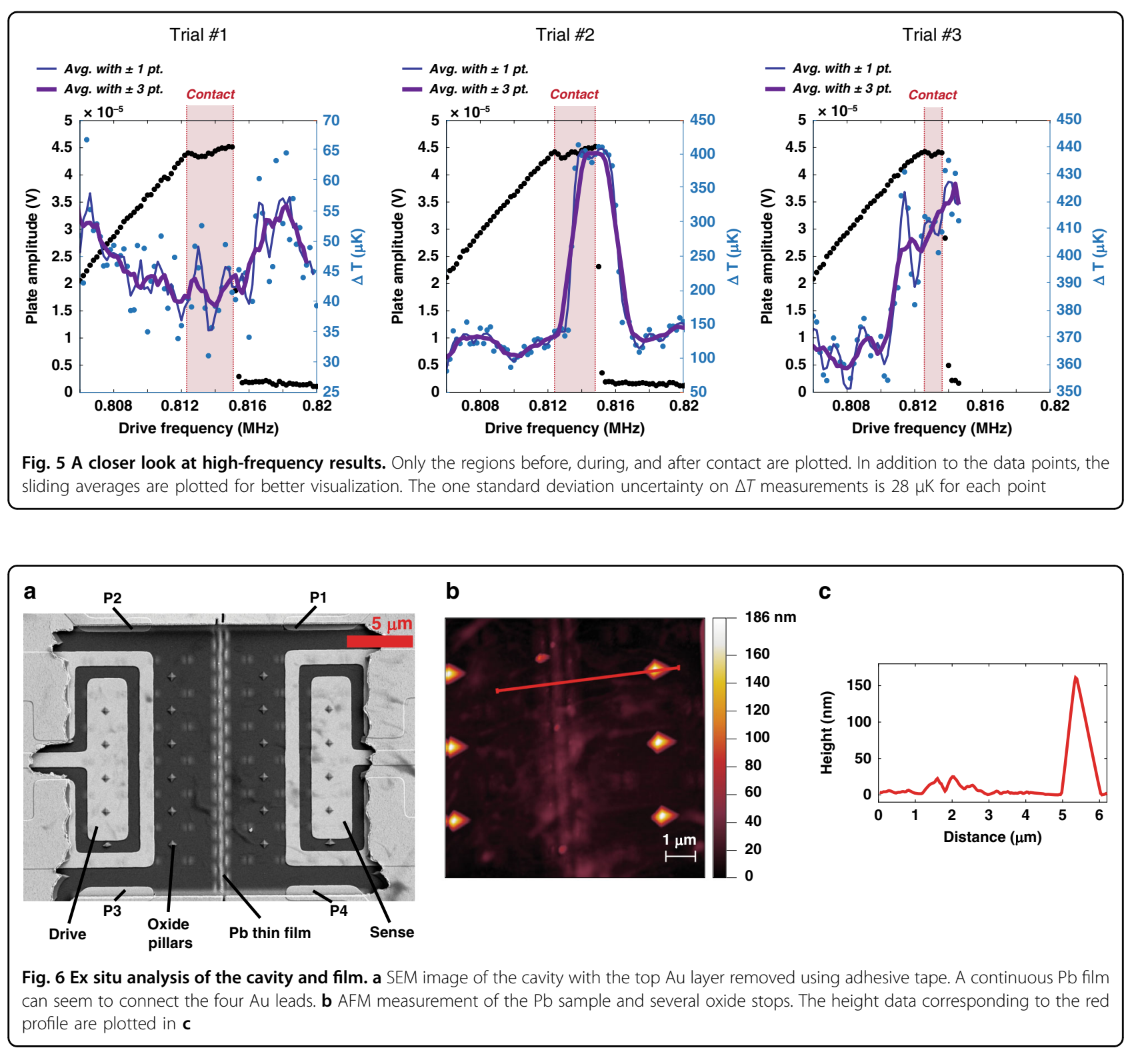

of the suspended Au layer at cryogenic temperature is unknown. Using a series of finite element simulations, a likely range of the minimum $\mathrm{Au} / \mathrm{Pb}$ spacing (i.e., when the amplitude of the plate is maximum) is estimated to be between 63 and $73 \mathrm{~nm}$. Across the entire length of the sample, the average $\mathrm{Au} / \mathrm{Pb}$ spacing at maximum amplitude is estimated to lie between 85 and $141 \mathrm{~nm}$. This analysis is explained further in the "Methods" section.

\section{Discussion}

We have developed a platform based on a variable gap cavity including an $\mathrm{Au}$ nanoscale mechanical resonator and a high-quality quenched-condensed superconductor film, enabling the simultaneous electromechanical control of the nanometric gap between plates and the transport measurement of the superconducting film using a fourprobe configuration. The high-frequency detection method presented makes use of the natural resonance of the plate to obtain the large modulations of the cavity size and allows for the phase-sensitive detection of changes in the sample resistance due to the plate motion only.

\section{Analysis of the results}

In this set of experiments, the temperature of the cryostat is set to the steepest part of the superconducting transition $(T \approx 6.88 \mathrm{~K})$, and three long-duration sweeps are made driving the plate to resonance from low to high frequency until it abruptly loses amplitude due to its nonlinear resonant response. This nonlinearity is well described by a simple Duffing equation with spring 
stiffening due to the mechanics of the doubly clamped plate. More general descriptions that include the electrostatic driving, roughness, or Casimir force ${ }^{27}$ are not required. An important feature in the plate response is the evidence in all three trials of a sudden clear deviation from the Duffing-nonlinear oscillator model in the amplitude and phase behavior occurring at $\sim 811.2 \mathrm{kHz}$. This finding is due to the onset of the plate interacting with the oxide stops. Any additional nonlinearities in the plate mechanics due to deformation alone does not appear in this discontinuous manner but instead likely appears gradually. In addition, the amplitude is nearly fixed beyond this point, which is a further indication of contact. Upon contacting the pillars, the maximum peak displacement of the most deflected point on the plate centerline is estimated to be between 183 and $193 \mathrm{~nm}$, resulting in a minimum Casimir cavity size of between 63 and $73 \mathrm{~nm}$. Along the entire length of the superconducting sample and plate center, we estimate the average gap size at the closest approach to be between 85 and $141 \mathrm{~nm}$. After the plate reaches its maximum amplitude at resonance $(\sim 815 \mathrm{kHz})$, the plate amplitude abruptly drops to zero, and the undeflected Casimir cavity size returns to $\approx 256 \mathrm{~nm}$. This jump is where we might expect to see more clearly a corresponding change in the sample resistance if there were indeed a dependence of the superconducting transition temperature on the cavity size; however, no such statistically significant correlation is observed.

Another feature of the high-frequency $\Delta T$ measurements is seemingly random jumps of (300-400) $\mu \mathrm{K}$. The cause of these instabilities is unknown; however, based on analyzing the three trials together, we do not believe they are related to the plate position. For example, in trial \#1, we observe a large displacement at $820 \mathrm{kHz}$, which is well after the large plate oscillations have ceased. In trial \#2, there is a large jump that is interestingly close to the minimum gap range, but a closer inspection of the data in Fig. 5 shows that it lags the plate contact by 4 or 5 data points (corresponding to 6-7.5 min of time difference), which is thus highly unlikely to be the effect we are looking for. Finally, in trial \#3, $\Delta T$ appears to be tracking the plate amplitude, but then after the oscillations jump down, the signal does not follow. While undesirable, it is reasonable to assume that these intermittent jumps did not obscure the correlation between the abrupt change in the plate amplitude and the sample measurement. Rather, it is the underlying stochastic noise, indicated by the spread of the data of the individual areas shown in the green boxes in Fig. 4, which determines the experimental resolution.

To accurately quantify the uncertainty of the temperature measurement, we first consider this stochastic spread of the raw data as well as the uncertainty in the slope of the transition. For this value, we use the lower $95 \%$ confidence bound of the slope $(11 \mathrm{k} \Omega / \mathrm{K})$ to conservatively claim a resolution. Using one standard deviation from each data set shown in the green areas of Fig. 4, averaging these with a weight prescribed by the number of data points in each set, and dividing by the lower estimate of the slope, we calculate one standard deviation per data point of $28 \mu \mathrm{K}$.

We look for the change in the critical temperature at the point of the abrupt change from a high amplitude vibration to the almost negligible vibration of the plate. At this jump, we quantify the change in the measured data by averaging 4 individual $\Delta T$ points immediately before and immediately after the jump and subtracting the averaged values. The average difference from the three experimental runs is $(7 \pm 12) \mu \mathrm{K}$. Thus, any effect of plate position on the transition temperature of the superconductor in our system is well below the one standard deviation statistical uncertainty of $12 \mu \mathrm{K}$.

\section{Interpretation of the experiment}

In all three trials, no clear correlation between the plate amplitude or position and transition temperature was observed above our measurement resolution of $28 \mu \mathrm{K}$, and from the three trials combined, no change in $T_{\mathrm{c}}$ was observed to exceed the one standard deviation uncertainty of $12 \mu \mathrm{K}$. There are a few reasons that may explain this null result. No observation of this effect may be due to one or more of the following: (1) geometrical limitations, (2) superconductor limitations, and (3) theoretical uncertainties.

In the first case, the device geometry may not allow for a sufficiently small cavity to clearly observe a shift. The minimum cavity size that we are able to reach with the current configuration is at best on the order of $70 \mathrm{~nm}$. Although this brings us into a theoretically interesting range, it may not be small enough to produce a measurable shift with the current materials. The uncertainty in the exact gap size exists due to the uncertain stress in the structure near $7 \mathrm{~K}$, which is expanded upon in the "Methods" section. Using a basic scaling law shown in Eq. (8) (derived from calculations performed in ref. ${ }^{18}$ ), we estimate the relative change in the Casimir-free energy between this minimum cavity size and the undeformed cavity size to be $\approx 400 \%$. Further optimization of the timed oxide undercut and geometric design may allow for smaller cavities, which increase the magnitude of the Casimir-free energy compared to the condensation energy of the $\mathrm{Pb}$ film. Additionally, as discussed in the "Methods" section, the resonant mode shape analysis of the Au plate indicates that there is likely some degree of bowing of the plate along the length of the sample at the time of the closest approach, reducing the parallelism of the Au and $\mathrm{Pb}$ surfaces and thus the overall Casimir interaction. 
Regarding the superconducting film itself, there are certain key material properties to consider when conducting this type of experiment. First, a low $T_{\mathrm{c}}$ value is generally desired because the condensation energy scales $\propto T_{\mathrm{c}}^{2.628}$. As discussed, it is the ratio of the Casimir-free energy to the condensation energy that determines the magnitude of the shift in the critical field, so generally speaking, the lower $T_{\mathrm{c}}$ is, the better. In the case of the experiment presented here, $\mathrm{Pb}$ has a relatively high $T_{\mathrm{c}}$ value but was chosen for other experimental advantages. Future work may involve investigating lower $T_{\mathrm{c}}$ materials. Another important material property is the plasma frequency. Bimonte et al. ${ }^{18}$ showed that high plasma frequency materials can change the strength of the Casimirfree energy term by almost one order of magnitude. Many of the calculations in their work use are, with a plasma frequency of $\sim 18 \mathrm{eV}$, which is higher than $\mathrm{Pb}$, with a value of $\sim 8 \mathrm{eV}^{29}$. This reduction in the reflectivity at higher frequencies may result in a Casimir energy contribution to the free energy of condensation that is too small to observe.

Finally, there is the question of what we expect theoretically in the limit of zero applied magnetic field. The calculation methods used in this low field limit are not possible due to the condensation energy and the change in the free energy becoming comparable ${ }^{30}$. It is therefore not exactly clear what one might expect in terms of the magnitude of the change in the critical temperature as a result of a Casimir energy variation. Our experiment shows that for our geometry, materials, and in the range of temperatures we can resolve, there is no observed effect. Most certainly, the next steps in this project will involve extending the experiment to include magnetic characterization and extending the existing theory to the $H=0$ case $^{31}$.

\section{Conclusions}

We have developed a unique nanomechanical transducerbased measurement technique and have undertaken a careful series of experiments to directly measure the shifts in the Casimir energy by placing a superconducting $\mathrm{Pb}$ film in a cavity and tuning the gap, looking for effects on the superconducting transition temperature of the film. Our chip-scale system can deposit and measure a superconducting thin film while simultaneously actuating a nearby plate, forming a tunable Casimir cavity. The in situ deposition process is achieved with two arrays of MEMS heaters that have been preloaded with a thick film of $\mathrm{Pb}$ and can be pulsed at low temperature to evaporate small amounts of material. The thin film is produced by using a shadow mask to define a precise pattern of the evaporated $\mathrm{Pb}$ (incident on the mask from two sides) that connects the four metallic measurement leads and creates a thin section of $\mathrm{Pb}$ directly underneath the movable Au plate. By driving a current with two sets of leads and measuring the voltage drop with the other two, we can measure the resistance of $\mathrm{Pb}$. We monitor this resistance as the Au plate is driven to its mechanical resonance and back to zero amplitude.

Using finite element analysis, we are able to estimate the deformed mode shape at resonance, which places the minimum separation of $\mathrm{Pb}$ and Au between 63 and $73 \mathrm{~nm}$. We estimate that at this gap size, the presence of the $\mathrm{Au}$ plate may be expected to produce the relative changes in the Casimir-free energy of the $\mathrm{Pb}$ film of $2 \times$ to $4 \times$; however, a quantitative prediction for the corresponding change in $T_{\mathrm{c}}$ is a subject of ongoing theoretical work ${ }^{31}$ and is not available at present. The results presented here, at zero applied magnetic field, indicate that there are no observed Casimir-induced changes in $T_{\mathrm{c}}>12 \mu \mathrm{K}$. By opening a novel experimental window regarding the relationship between the Casimir energy and superconductivity, we hope this work will stimulate further experimental and theoretical developments.

\section{Methods \\ In situ film deposition and characterization}

An experiment is performed in a closed-cycle cryostat specially designed for low mechanical vibrations. A typical experiment is performed as follows: (1) The source and target are mounted opposite one another and cooled to $\approx 3 \mathrm{~K}$ in the closed cycle system, (2) at low temperatures, $\mathrm{Pb}$ is evaporated onto the substrate, producing a smooth, continuous superconducting film, (3) the resistance of the $\mathrm{Pb}$ film is measured across its transition, and (4) the spacing of the Casimir cavity is modulated by applying an alternating voltage between the drive electrode and the suspended $\mathrm{Au}$ plate while the temperature of the system is held at the shoulder of the superconducting transition.

The resistance and transition of the $\mathrm{Pb}$ film are initially measured using a four-point configuration, shown schematically in Fig. 3 (inset). An excitation current, $I_{\mathrm{ex}}$, is applied at lead $I+$ (in series with an external $1 \mathrm{M} \Omega$ resistor) and travels through the central portion of the sample to $I-$, which is grounded through a $1 \mathrm{k} \Omega$ resistor. A voltage difference is then measured between the other two leads, $V+$ and $V-$. $I_{\text {ex }}$ is a low-frequency AC current of $\approx 5 \mathrm{nA}$ at $37.7 \mathrm{~Hz}$. The differential voltage measurement is measured using a lock-in amplifier, and the resistance of the sample is then $V_{\mathrm{diff}} / I_{\mathrm{ex}}$. The temperature of the system is slowly swept across the transition (both down and up), which provides the slope of the transition, $\mathrm{d} R_{\mathrm{s}} / \mathrm{d} T$, as well as $T_{\mathrm{c}}$.

\section{Plate actuation and high-frequency measurement}

The dynamic detection scheme involves measuring $V_{\text {diff }}$ at the same frequency the plate is moving. First, we set the cryostat temperature to just below $T_{\mathrm{c}}$, where the 
resistance measurement is the most sensitive to the changes in temperature. Next, if the position of the plate does indeed influence the sample resistance due to the Casimir effect, then we expect a small modulation of $R_{\mathrm{s}}$ at the plate frequency. Subsequently, the voltage difference measured is as follows:

$$
V_{\text {diff }}=I_{\mathrm{ex}} \cdot\left(R_{\mathrm{s}, 0}+\Delta R(t)\right)
$$

where $R_{\mathrm{s}, 0}$ is the nominal sample resistance and $\Delta R(t)$ is the small variation due to the plate. We can further breakdown $\Delta R$ :

$$
\Delta R=\underbrace{\frac{\partial R}{\partial T}}_{\text {transition slope }} \cdot \underbrace{\frac{\partial T}{\partial d}}_{\text {Casimir }} \cdot \underbrace{\mathrm{d}(t)}_{\text {plate position }}
$$

The "Casimir" term is the theorized change in $T_{\mathrm{c}}$ of the $\mathrm{Pb}$ sample due to the changing position of the plate, $\mathrm{d}(t)$. This change is what we intend to detect. Thus, if we consider only the component of $V_{\text {diff }}$ at the plate frequency, with amplitude $\left|V_{\text {diff }}\right|_{\text {plate }}$, then we can rearrange equation $\Delta R$ to obtain the amplitude of this expected effect:

$$
\left|\frac{\partial T}{\partial d}\right|=\frac{\left|V_{\text {diff }}\right|_{\text {plate }}}{\left|I_{\text {ex }}(t)\right| \cdot \frac{\partial R}{\partial T} \cdot|\mathrm{d}(t)|}
$$

In this case, a more complex detection circuit is required. In Fig. 7, a high-frequency $\mathrm{AC}$ drive signal is used to actuate the plate at its resonance. The plate amplitude is detected at twice this drive frequency using LIA 1 . Simultaneously, a current goes through the sample at a low frequency, while $V_{\text {diff }}$ is being measured at the same frequency as the plate using LIA 2. The output of LIA 2 is then fed into LIA 3, which is locked into the low frequency of the excitation signal. The DC output of LIA 3 is then equal to the amplitude $\left|V_{\text {diff }}\right|_{\text {plate. }}$. The inset table in Fig. 7 reports nominal values of each parameter presented in the schematic.

\section{Variation in the Casimir-free energy due to plate motion}

It is possible to estimate the relative variation in the Casimir-free energy difference using the result from ref. ${ }^{18}$

$$
\Delta E_{\text {Cas }} \propto \frac{1}{1+\left(\frac{d}{d_{0}}\right)^{1.15}}
$$

where $d$ is the separation between the superconductor and metal and $d_{0}$ is the nominal separation $(\approx 256 \mathrm{~nm}$ in our experiment). It should be noted, however, that ref. ${ }^{18}$ approximates the relation shown in Eq. (6) using a system, where $T_{\mathrm{c}}=0.5 \mathrm{~K}$, the superconductor thickness is $5 \mathrm{~nm}$, and $d_{0}=8.3 \mathrm{~nm}$, which is different than the system presented here. Considering the relative change in $\Delta E_{\mathrm{Cas}}$ to be $\delta E_{\text {Cas }}=\Delta E_{\text {Cas }}(d) / \Delta E_{\text {Cas }}\left(d_{0}\right)$ with $\mathrm{d}(t)$ changing sinusoidally at $\omega=2 \pi f_{2}$ with amplitude $A$, we find:

$$
\delta E_{\text {Cas }}(t, A)=\frac{2}{1+\left(\frac{d_{0}+A \sin (\omega t)}{d_{0}}\right)^{1.15}}
$$

To a first approximation, we assume that $\delta E_{\text {Cas }}(t)$ varies $T_{\mathrm{c}}(t)$ linearly. Because the phase-sensitive detection used in the experiment only measures the time-averaged component of the signal at $\omega$, we can approximate Eq. (7) by considering only the time-averaged magnitude of the first harmonic term as a function of $A$. Numerically solving for the magnitude of the first Fourier coefficient of $\delta E_{\text {Cas }}(t, A)$ over the range $A=0-200 \mathrm{~nm}$ and using $d_{0}=$ $256 \mathrm{~nm}$, we find that it can be well approximated by a third-order polynomial:

$$
\left|\delta E_{\text {Cas }}\right|=c_{1} A+c_{2} A^{3}
$$

where $c_{1}=0.0225 \mathrm{~nm}^{-1}$ and $c_{2}=5.905 \times 10^{-9} \mathrm{~nm}^{-3}$. Using Eq. (8), we can then estimate the relative change in the Casimir-free energy for a given plate amplitude. This estimate considers two parallel areas, separated by $d$. In reality, the interaction of the $\mathrm{Au}$ plate and $\mathrm{Pb}$ sample is not parallel, and the geometry is defined by the deformed shape of the plate. An exact analytical calculation of the Casimir interactions between general, nonparallel shapes is an open problem, which we do not address in this analysis. However, a study of how the plate might be deforming at its resonance is discussed in the following section.

\section{Estimating the plate deflection and mode shape using finite element analysis}

Determining the exact amplitude of the plate and therefore the Casimir gap size is challenging due to a significant change in the dynamics that occurs between room temperature and the cryogenic temperature. This behavior is demonstrated by a large change in the resonant frequency, from $\approx 700 \mathrm{kHz}$ to $\approx 1.8 \mathrm{MHz}$ between 300 and $3 \mathrm{~K}$, respectively, indicating the substantial tensile stress that occurs in the top $\mathrm{Au}$ layer due to thermal contraction of the Au relative to the substrate. By examining the behavior of the signal at the sense electrode, it is possible to determine when contact with the oxide pillars occurs; however, determining the distance between the $\mathrm{Pb}$ sample and the center of the plate (parallel to the $\mathrm{Pb}$ sample) requires knowledge of the deformed shape of the $\mathrm{Au}$ plate at its resonance.

Qualitatively, a Duffing response nonlinearity arises due to the dynamic increase in the tension of the vibrating $\mathrm{Au}$ plate, which slightly increases the mean 


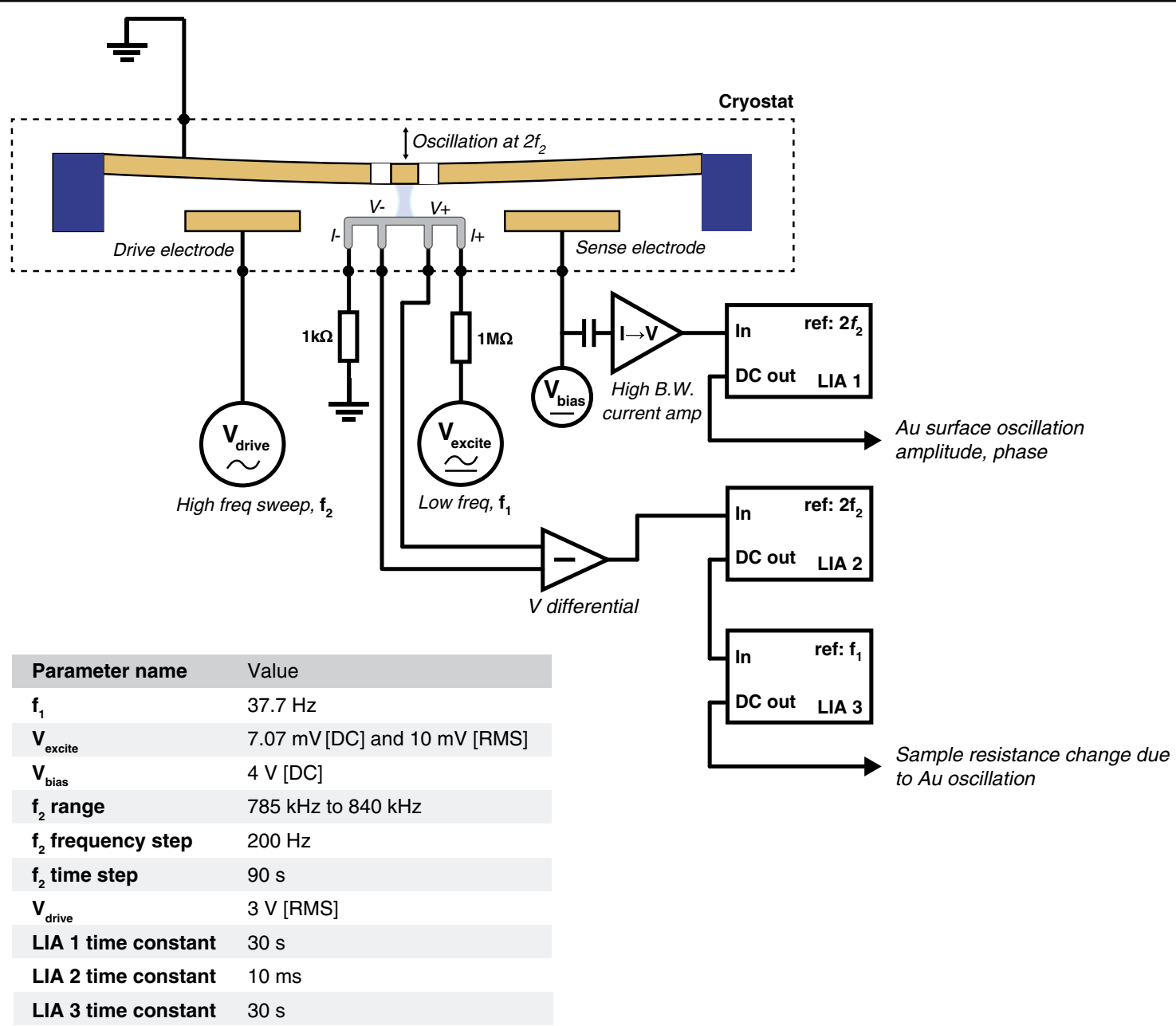

Fig. 7 High-frequency detection circuit. The cryostat is held at a constant temperature just below $T_{c}$ on the slope of the transition. The highfrequency $V_{\text {drive }}$ signal is applied to the drive electrode and swept at frequency $f_{2}$. The plate then feels an electrostatic force at frequency $2 f_{2}$. The amplitude of the plate is monitored by measuring the AC current going through the sense electrode using a current-to-voltage amplifier and a lockin referenced to $2 f_{2}$ (LIA 1). The voltage drop across the Pb sample is also detected at $2 f_{2}$. If there is any change in $T_{c}$ due to the Casimir cavity size, then this is the frequency at which it would occur. Because the excitation current, $l_{\text {ex, }}$, is alternating at $f_{1}$, the DC output of LIA 2 is fed into a third lockin, LIA 3, which is referenced at $f_{1}$

resonance frequency with amplitude. However, because this change in the dynamic stress over an oscillation cycle is much smaller than the static stress, the mode shape is not much different than that of a linear, prestressed structure. Using a commercial finite element software package to calculate mechanical eigenmodes, three different initial stress cases are considered to place bounds on the distance at which the center of the plate deflects at resonance. The first case considers zero initial stress, and the second and third cases include two different values of a uniaxially applied initial stress ( 0.1 and $0.26 \mathrm{GPa}$, respectively).

After a mode shape is obtained, it is scaled in amplitude $(Z)$ until any part of the surface comes into contact with any of the oxide pillars, and then the profile parallel to the sample is extracted. In Fig. 8a, the values of maximum separation, minimum separation, and average separation are reported as well as the two lengths: $\mathrm{L}_{10}$ and $\mathrm{L}_{25}$, which are the lengths of the sample that are within $10 \%$ and $25 \%$, respectively, of the minimum gap size.

Case 1: Although there is clearly evidence of significant initial stress in the movable Au layer, it is necessary to estimate the upper bound of the plate deflection by considering the zero stress case. Solving for the first eigenfrequency of the device geometry returns a value of $470 \mathrm{kHz}$, which is, as expected, well below the measured $1.8 \mathrm{MHz}$.

Case 2: Clearly, for the simulation to match the measured resonant frequency, a prestress must be applied. The reason for the two different stress cases 2 and 3 is 
a

\begin{tabular}{|l|c|c|c|c|c|}
\cline { 2 - 6 } \multicolumn{1}{c|}{} & Min. gap & Max. gap & Avg. gap & $\boldsymbol{L}_{10}$ & $\mathbf{L}_{25}$ \\
\hline Case 1 & $63 \mathrm{~nm}$ & $93 \mathrm{~nm}$ & $85 \mathrm{~nm}$ & $1.2 \mu \mathrm{m}$ & $4.6 \mu \mathrm{m}$ \\
\hline Case 2 & $73 \mathrm{~nm}$ & $131 \mathrm{~nm}$ & $118 \mathrm{~nm}$ & $0.8 \mu \mathrm{m}$ & $2.0 \mu \mathrm{m}$ \\
\hline Case 3 & $66 \mathrm{~nm}$ & $169 \mathrm{~nm}$ & $141 \mathrm{~nm}$ & $0.5 \mu \mathrm{m}$ & $1.3 \mu \mathrm{m}$ \\
\hline
\end{tabular}

b

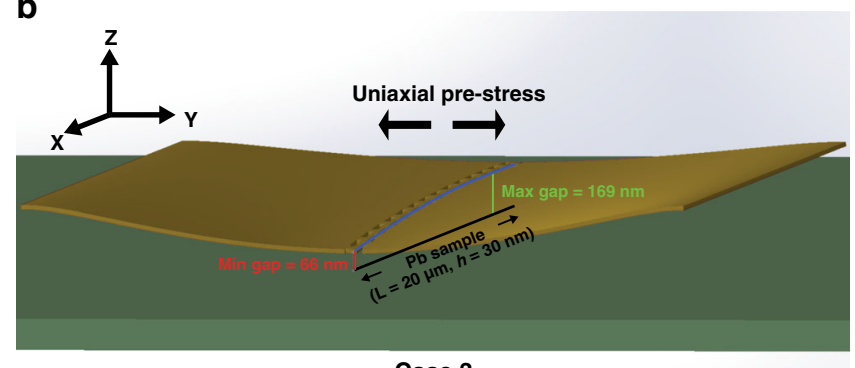

Case 3

Fig. 8 Finite element analysis for determining the mode shape and cavity size. a Results from the finite element analysis for gap sizes achieved along the length of the $\mathrm{Pb}$ sample (20 $\mu \mathrm{m}$ in length) upon contact between the deformed Au plate and the oxide pillars. With no actuation, the gap size is assumed to be $256 \mathrm{~nm}$ (sample thickness subtracted from oxide thickness). $\mathbf{b}$ 3D representation of the gap size estimation using finite element results for case 3 (high uniaxial stress). In this case, the profile of the plate along the sample (blue line) is asymmetric and reaches much closer to the sample on one side compared to the other. This reduces the average gap size as well as $L_{10}$ and $L_{25}$

that in the high-stress limit, finite element analysis suggests that the fundamental mode and the second mode become near degenerate and mix due to the slight layout asymmetry in the center cut of the moving plate. This resulting high-stress mode shape is essentially only one side of the cavity moving up and down, while the other side moves with a much smaller amplitude. Case 2 considers an intermediate stress case before this degeneracy is reached, and the shape of the fundamental mode still resembles that of the zero stress case. In this simulation, the uniaxial prestress is equal to $0.1 \mathrm{GPa}$, and the resulting resonant frequency is $1.12 \mathrm{MHz}$.

Case 3: In this simulation, the uniaxial prestress is increased until we reach a resonant frequency that matches the experiment $(\approx 1.8 \mathrm{MHz})$. This stress is equal to $0.26 \mathrm{GPa}$ and is generally consistent with the estimates from the differential thermal expansion. However, in this high-stress condition, the mode shape is very asymmetric due to the mixing of the fundamental and the second modes. As a result, only one edge of the Au displaces enough to become close to the sample (Fig. 8b).

A full understanding of the exact shape of the Au plate at its resonance is not possible; however, by using the measured current amplitude from the experiment in conjunction with post-experimental finite element analysis, we can infer that the minimum separation of the $\mathrm{Pb}$ sample and the Au plate is likely between 63 and $73 \mathrm{~nm}$ and that the average separation across the length of the sample is likely between 85 and $141 \mathrm{~nm}$. Using the estimated scaling dependence shown in Eq. (8), these deflections would produce relative changes in the
Casimir-free energy in the $\mathrm{Pb}$ sample between $415 \%$ and $439 \%$ (when considering only the area near the minimum gap) and between $260 \%$ and $388 \%$ when considering the average gap over the length of the sample.

\section{Acknowledgements}

We would like to thank Daron Westly, Mark D. Stiles, Ph.D., J. Alexander Little, Ph.D., and Marcelo Davanco, Ph.D. for reviewing this manuscript and offering many meaningful suggestions. Additional thanks to Fartash Yusefi for the collection of the AFM data. This work is supported in part by the Cooperative Research Agreement between the University of Maryland and the National Institute of Standards and Technology Center for Nanoscale Science and Technology, Award 70NANB10H193, through the University of Maryland. Additional support of this work is provided by the National Science Foundation under Grants EEC-1647837, ECCS-1708283, EEC-0812056, a SONY Faculty Innovation Award, and DARPA/AFRL through award FA8650-15-C-7545.

\section{Author details}

${ }^{1}$ Department of ECE, Boston University, Boston, MA 02215, USA. ${ }^{2}$ Physical Measurement Laboratory, National Institute of Standards and Technology, Gaithersburg, MD 20899, USA. ${ }^{3}$ Institute for Research in Electronics and Applied Physics \& Maryland NanoCenter, University of Maryland, College Park, MD 20742, USA. ${ }^{4}$ Division of MSE, Boston University, Boston, MA 02215, .USA ${ }^{5}$ 4K-MEMS Sàrl, Rue des Lavannes 17, 2072 St Blaise, Switzerland. ${ }^{6}$ Department of Physics, Boston University, Boston, MA 02215, USA. ${ }^{7}$ Department of ME, Boston University, Boston, MA 02215, USA. ${ }^{8}$ Department of BME, Boston University, Boston, MA 02215, USA

\section{Author contributions}

The device design and experiments were conceived by D.P.-M., V.A.K., and D.J.B. Target device fabrication was carried out by D.P.-M. Microsource device design and preparation was conducted by D.P.-M. and A.S. with input from R.L. Measurement techniques were developed by D.P.-M., A.S., M.I., V.A.K., and D.J.B. with experiments performed by A.S. The analysis of the data and finite element modeling was performed by A.S. with input from V.A.K. and D.P.-M. The manuscript was written by A.S. with input from D.P.-M., V.A.K., L.K.B., M.I., R.L., A. Som, D.K.C., and D.J.B. 


\section{Conflict of interest}

The authors declare that they have no conflict of interest.

Supplementary information accompanies this paper at https://doi.org/ 10.1038/s41378-020-00221-2.

Received: 4 May 2020 Revised: 21 September 2020 Accepted: 19 October 2020

Published online: 28 December 2020

\section{References}

1. Casimir, H. B. On the attraction between two perfectly conducting plates. Proc. K. Ned. Akad. Wet. 51, 150 (1948)

2. Lamoreaux, S. K. Demonstration of the Casimir force in the 0.6 to $6 \mu \mathrm{m}$ range. Phys. Rev. Lett. 78, 5-8 (1997).

3. Mohideen, U. \& Roy, A. Precision measurement of the Casimir force from 0.1 to $0.9 \mu \mathrm{m}$. Phys. Rev. Lett. 81, 4549 (1998).

4. Chan, H. B., Aksyuk, V. A., Kleiman, R. N., Bishop, D. J. \& Capasso, F. Quantum mechanical actuation of microelectromechanical systems by the Casimir force. Science 291, 1941-1944 (2001)

5. Chan, H. B., Aksyuk, V. A., Kleiman, R. N., Bishop, D. J. \& Capasso, F. Nonlinear micromechanical Casimir oscillator. Phys. Rev. Lett. 87, 211801 (2001).

6. Tang, L. et al. Measurement of non-monotonic Casimir forces between silicon nanostructures. Nat. Photonics 11, 97-101 (2017)

7. Antezza, M. et al. Giant Casimir torque between rotated gratings and the $\theta=0$ anomaly. Phys. Rev. Lett. 124, 013903 (2020).

8. Stange, A., Imboden, M., Javor, J., Barrett, L. \& Bishop, D. J. Building a Casimir metrology platform with a commercial MEMS sensor. Microsyst. Nanoeng. 5, 14 (2019)

9. Jourdan, G., Lambrecht, A., Comin, F. \& Chevrier, J. Quantitative non-contact dynamic Casimir force measurements. EPL 85, 31001 (2009).

10. Imboden, M., Morrison, J., Campbell, D. \& Bishop, D. Design of a Casimir-driven parametric amplifier. J. Appl. Phys. 116, 134504 (2014).

11. Munday, J., Capasso, F. \& Parsegian, V. Measured long-range repulsive Casimir-Lifshitz forces. Nature 457, 170-173 (2009)

12. Levin, M., McCauley, A. P., Rodriguez, A. W., Reid, M. T. H. \& Johnson, S. G. Casimir repulsion between metallic objects in vacuum. Phys. Rev. Lett. 105, 090403 (2010)
13. Intravaia, F. et al. Strong Casimir force reduction through metallic surface nanostructuring. Nat. Commun. 4, 2515 (2013).

14. Bimonte, G., Lopez, D. \& Decca, R. Isoelectronic determination of the thermal Casimir force. Phys. Rev. B93, 184434 (2016).

15. Glover, R. E. \& Tinkham, M. Conductivity of superconducting films for photon energies between 0.3 and $40 \mathrm{kT}$. Phys. Rev. 108, 243 (1957).

16. Norte, R. A., Forsch, M., Wallucks, A., Marinković, I. \& Gröblacher, S. Platform for measurements of the Casimir force between two superconductors. Phys. Rev. Lett. 121, 030405 (2018).

17. Bimonte, G., Calloni, E., Esposito, G., Milano, L. \& Rosa, L. Towards measuring variations of Casimir energy by a superconducting cavity. Phys. Rev. Lett. 94, 180402 (2005).

18. Bimonte, G., Calloni, E., Esposito, G. \& Rosa, L. Variations of Casimir energy from a superconducting transition. Nucl. Phys. B 726, 441-463 (2005).

19. Neugebauer, C. A. \& Webb, M. B. Electrical conduction mechanism in ultrathin, evaporated metal films. J. Appl. Phys. 33, 74-82 (1962).

20. Ekinci, K. L. \& Valles, J. M. Jr. Morphology of quench condensed Pb Films near the insulator to metal transition. Phys. Rev. Lett. 82, 1518 (1999).

21. Imboden, M., Han, H., Stark, T. \& Bishop, D. Cryogenic fab on a chip sticks the landing. ACS Nano 11, 20274-20285 (2017).

22. Imboden, M. et al. Atomic calligraphy: the direct writing of nanoscale structures using MEMS. Nano Lett. 13, 3379-3384 (2013).

23. Imboden, M. et al. Building a fab on a chip. Nanoscale 6, 5049-5062 (2014).

24. Imboden, M. \& Bishop, D. Top-down nanomanufacturing. Phys. Today 67, 45-50 (2014)

25. Han, $\mathrm{H}$. et al. Programmable solid state atom sources for nanofabrication Nanoscale 7, 10735-10744 (2015).

26. Franck, J. P. \& Martin, D. L. The superconducting transition temperature of lead. Can. J. Phys. 39, 1320-1329 (1961).

27. Broer, W., Waalkens, H., Svetovoy, V. B., Knoester, J. \& Palasantzas, G. Nonlinear actuation dynamics of driven casimir oscillators with rough surfaces. Phys. Rev. Appl. 4, 054016 (2015).

28. Lewis, H. W. Superconductivity and electronic specific heat. Phys. Rev. 101 939-939 (1956).

29. Ordal, M. A., Bell, R. J., Alexander, R. W., Long, L. L. \& Querry, M. R. Optical properties of fourteen metals in the infrared and far infrared: $\mathrm{Al}, \mathrm{Co}, \mathrm{Cu}, \mathrm{Au}, \mathrm{Fe}$, Pb, Mo, Ni, Pd, Pt, Ag, Ti, V, and W. Appl. Opt. 24, 4493 (1985).

30. Bimonte, $G$. et al. Low noise cryogenic system for the measurement of the Casimir energy in rigid cavities. J. Phys. A 41, 164023 (2008).

31. Som, A., Aksyuk, V., Barrett, L. K., Imboden, M., Lally, R. K., Pérez-Morelo, D. Stange, A., Bishop, D. J. \& Campbell, D. K. On-going work (2020). 